\title{
Direction-of-Arrival Estimation of Closely Spaced Emitters Using Compact Arrays
}

\author{
Hoi-Shun Lui ${ }^{1,2}$ and Hon Tat Hui \\ ${ }^{1}$ Department of Signals and Systems, Chalmers University of Technology, 41296 Gothenburg, Sweden \\ ${ }^{2}$ School of Information Technology and Electrical Engineering, The University of Queensland, Brisbane, QLD 4072, Australia \\ ${ }^{3}$ Department of Electrical and Computer Engineering, National University of Singapore, Singapore 117576
}

Correspondence should be addressed to Hoi-Shun Lui; h.lui@uq.edu.au

Received 14 May 2012; Accepted 1 November 2012

Academic Editor: Charles Bunting

Copyright (c) 2013 H.-S. Lui and H. T. Hui. This is an open access article distributed under the Creative Commons Attribution License, which permits unrestricted use, distribution, and reproduction in any medium, provided the original work is properly cited.

Performance evaluation of direction-of-arrival (DOA) estimation algorithms has continuously drawn significant attention in the past years. Most previous studies were conducted under the situation that antenna element separation is about half wavelength in order to avoid the appearance of grating lobes. On the other hand, recent developments in wireless communications have favoured the use of portable devices that utilize compact arrays with antenna element separations of less than half wavelength. Performance evaluation of DOA estimation algorithms employing compact arrays is an important and fundamental issue, but it has not been fully studied. In this paper, the performance of the matrix pencil method (MPM) that applies to DOA estimations is investigated through Monte Carlo simulations. The results show that closely spaced emitters can be accurately resolved using linear compact array with an array aperture as small as around half wavelength.

\section{Introduction}

Direction-of-arrival (DOA) estimation is one of the most important applications in array signal processing. In the context of array signal processing, these algorithms can be grouped into three categories: conventional methods, subspace methods, and maximum likelihood techniques [1-4]. Throughout the last two decades, subspace methods have achieved great success due to their low complexity and reasonable performance. Out of all the subspace methods, Pisarenko's method [4] was one of the first methods that exploited the structure of the data model. Schmidt [5] then studied the signal properties of a sensor array and introduced the Multiple Signal Classification (MUSIC) algorithm. The MUSIC algorithm exploits the geometrical structure of the signal. Performance evaluations of the MUSIC algorithm have been well studied in the 1980s to mid-1990s, for example [6,7]. The main advantage of subspace methods over the conventional beamforming method is that the resolution is not limited by the aperture of the entire array, and subspace methods are therefore also known as super resolution techniques [8].
Another well-known subspace method is Estimation of Signal Parameters via Rotational Invariance Techniques (ESPRIT) $[9,10]$, which exploits the array structure such that the specific knowledge of the array manifold is not required. Compared to MUSIC, it is computationally more efficient without the need of searching peaks throughout the entire range of angles. A review about subspace methods from the statistical prospective can be found in [4]. In addition, there is another subspace method, known as the matrix pencil method (MPM), which was first applied to damped exponential extractions from transient electromagnetic signals [1113] and later to DOA estimations [14-20]. One significant advantage of MPM over MUSIC and ESPRIT is that DOA estimation can be done using a single snapshot instead of the covariance matrix that requires a large number of snapshots $[15,16]$. The incoming directions can be determined directly without the need for searching among all the directions as in the MUSIC. Even with coherent signals, spatial smoothing is not required.

The DOA estimation problem is about estimation of undamped exponentials based on the measured samples of 
the signal from an array. Performance evaluations of subspace methods and the corresponding Cramer-Rao Lower Bound (CRLB), which gives the lower bound on the variance of any unbiased estimator, have been well studied for the estimation of a single damped/undamped exponential (e.g., [21-28]). Attempts have also been made for the case with two exponentials embedded in the signal. For instance, Stoica and Nehorai [26] have studied the DOA estimation performance of MUSIC for the case of two equal powered signals, and the results showed that the variance of MUSIC algorithm is smaller when (i) the number of sensors (the length of data) increases, (ii) the signals are not highly correlated, and (iii) the two signals are not closely spaced. Steedly and Moses [27] derived the complete expressions for the CRLB of the parameters of an exponential model with one set of damped/ undamped exponentials and multiple sets of amplitude coefficients. For the case of two exponentials, numerical examples have shown that the angle CRLB is lower as the data length is large enough, and the signals are not closely spaced. In these studies, multiple snapshots of the signals or a single snapshot with long data length [27] are considered, and the separation between the sensors is usually fixed to the half wavelength. This is equivalent to the case where the array aperture is large (more than a wavelength if more than 3 elements). Such arrays are sometimes known as "standard arrays." The half wavelength separation is essentially the upper limit of Nyquist sampling rate [4] that avoids the appearance of grating lobes in the visible region of an array. At the same time, it provides the largest possible array aperture potentially resulting in the highest resolution, for instance, in conventional beamformers [1]. In addition, another practical consideration is the mutual coupling effect between antenna elements. In most array signal processing algorithms, the algorithms are developed under the assumption that interactions between array elements can be ignored. Mutual coupling is usually treated as a source of noise. Calibrations and preprocessing procedures are required to compensate such an undesirable effect [2931]. Recent work has demonstrated that the undesirable mutual coupling effect can be effectively compensated for arrays down to $\approx 0.1 \lambda$, both numerically $[32,33]$ and experimentally [34].

In the last two decades, there has been significant interest in reducing the physical dimensions of existing electronic devices with the rapid development of the electronics industry. In line with this development, recent applications in antenna arrays have been focused on compact array design [35-37] with less than a half wavelength element separation. To our knowledge, the performance of subspace methods under the situations where (i) the array aperture is significantly reduced to less than a wavelength and (ii) the increment of the number of sensors is within such a small array aperture has not been well exploited. Perturbation analysis for subspace methods and the corresponding CRLB are well studied for the single exponential process. When there is more than one mode embedded in the signals, however, the variance [21] and the CRLB [27] of the estimation problem are given in the form of matrix expressions. The results of how array aperture and sampling rates change can affect the performance of subspace methods, however, cannot be easily observed.

In view of this, this paper aims to characterize the effect of (i) changes in the array aperture with a fixed number of elements and (ii) changes in the number of elements in a fixed aperture on the accuracies DOA estimations with signals corrupted by Additive Gaussian White Noise (AGWN) using a Monte Carlo approach. In particular, the MPM will be considered. The focus of this study is on the effect of variations of the array aperture on the MPM algorithm under the ideal sensor condition. A brief review of MPM is first given followed by the Monte Carlo simulations and discussions towards the end of the paper.

\section{Matrix Pencil Method (MPM) for DOA Estimation}

Consider a space where there are $P+1$ signals coming from different directions and a uniform linear array (ULA) of $N+1$ identical sensors. The sensors are located along the $x$-axis with element separation between adjacent sensors denoted by $\Delta$. Then, the signal $x(n)$ received by the sensors can be written as $[11,14-22]$

$$
x(n)=\sum_{p=0}^{P} A_{p} \exp \left(j \gamma_{p}+\frac{j 2 \pi \Delta n \cos \phi_{p}}{\lambda}\right), \quad 0 \leq n \leq N
$$

where $A_{p}, \gamma_{p}$, and $\phi_{p}$ are, respectively, the amplitude, phase, and DOA of each of the $P+1$ plane wave sources incident on the ULA. $\lambda$ is the wavelength of the operation frequency. Equation (1) can also be written as

$$
x(n)=\sum_{p=0}^{P} c_{p} y_{p}^{n}
$$

where

$$
\begin{gathered}
c_{p}=A_{p} \exp \left(j \gamma_{p}\right), \\
y_{p}=\exp \left[\frac{\left(j 2 \pi \Delta \cos \phi_{p}\right)}{\lambda}\right] .
\end{gathered}
$$

In matrix form, this can be written as

$$
\left[\begin{array}{c}
x(0) \\
x(1) \\
\vdots \\
x(N)
\end{array}\right]=\left[\begin{array}{cccc}
1 & 1 & \ldots & 1 \\
y_{0} & y_{1} & \ldots & y_{p} \\
\vdots & \vdots & \ddots & \vdots \\
y_{0}^{N} & y_{1}^{N} & \cdots & y_{p}^{N}
\end{array}\right]\left[\begin{array}{c}
c_{0} \\
c_{1} \\
\vdots \\
c_{p}
\end{array}\right]
$$

or

$$
\mathbf{x}=\mathbf{Y c}
$$


Next, we define the $L \times(N-L+2)$ matrix $\mathbf{X}^{\prime}$ using $x(k)$, the elements of $\mathbf{X}$,

$$
\mathbf{X}^{\prime}=\left[\begin{array}{cccc}
x(0) & x(1) & \cdots & x(N-L+1) \\
x(1) & x(2) & \cdots & x(N-L+2) \\
\vdots & \vdots & \ddots & \vdots \\
x(L-1) & x(L) & \cdots & x(N)
\end{array}\right],
$$

where $L$ is the pencil parameter which satisfies the criteria of $(N+1-P) \geq L \geq(P+2)$. Applying a singular value decomposition (SVD) to $\mathbf{X}^{\prime}$, we have

$$
\mathbf{X}^{\prime}=\mathbf{U} \mathbf{\Sigma} \mathbf{V}^{H} \text {. }
$$

The superscript " $H$ " denotes the complex conjugate transpose of a matrix. If the data is not contaminated by noise, the first $P+1$ singular values are nonzero, and, hence, $P$ can be determined. If the data is noise contaminated, the parameter $P$ is estimated by observing the ratio of the various singular values to the largest one as defined by [11]

$$
\frac{\sigma_{c}}{\sigma_{\max }} \approx 10^{-w},
$$

where $w$ is the number of accurate significant decimal digits of the data $x(n)$. The singular values for which the ratio in (5) is below $10^{-w}$ are essentially noise singular values, and they should be discarded. It should be noted that when the incoming signals are spatially well resolved, these results in larger singular values are easier to be solved. For instance, if we have two cases with incoming signals from the directions of (i) $5^{\circ}$ and $6^{\circ}$ and (ii) $5^{\circ}$ and $46^{\circ}$, the second singular value in case (ii) is larger than that in case (i). As a result, only the cases of closely spaced emitters are considered in this work. The proposition here is that if we could find out how MPM performs under various array configurations for closely spaced emitters, it should also be able to work for spatially wellresolved signals.

Once $P$ is determined based on the condition given in (5), the submatrix of $\mathbf{U}$ with the first $P+1$ columns can be formed and denoted as $\mathbf{U}_{s}$. To estimate the DOAs, the following matrix pencil problem is solved:

$$
\mathbf{U}_{2}-\lambda^{\prime} \mathbf{U}_{1}=0 \text {, }
$$

or equivalently,

$$
\mathbf{U}_{1}{ }^{+} \mathbf{U}_{2}-\lambda^{\prime} \mathbf{I}=0,
$$

where $\mathbf{U}_{1}$ is $\mathbf{U}_{s}$ with the last row deleted, $\mathbf{U}_{2}$ is $\mathbf{U}_{s}$ with the first column deleted, $\lambda^{\prime}$ is the eigenvalue of the matrix pencil problem, $\mathbf{I}$ is the $(P+1) \times(P+1)$ identity matrix, and the superscript " + " denotes the Moore-Penrose pseudoinverse of a matrix [21] defined as

$$
\mathbf{U}_{1}^{+}=\left\{\mathbf{U}_{1}{ }^{H} \mathbf{U}_{1}\right\}^{-1} \mathbf{U}_{1}{ }^{H} .
$$

The superscript " -1 " denotes the inverse of a matrix. Once (6a), (6b), and (6c) are solved, the DOAs can be obtained by the following formula $[16,18]$ :

$$
\phi_{p}=\cos ^{-1}\left[\frac{\lambda \ln \left(\lambda_{p}{ }^{\prime}\right)}{j 2 \pi \Delta}\right],
$$

where $p=1,2, \ldots, P+1(P+1$ singular values from (6a), $(6 \mathrm{~b})$ and $(6 \mathrm{c}))$. It is well known that if the argument of the function $\cos ^{-1}$ in (7) is greater than 1 or a complex number, the resultant angle will become complex which is considered as a fail estimate for MPM. The resultant complex estimation angle can be used as a sanity check which indicates that MPM fails to resolve all the signals in that particular snapshot.

\section{Monte Carlo Simulations}

This section presents Monte Carlo simulations of DOA estimations using ULAs with different array apertures and different number of elements. Three cases are presented to illustrate our findings. First, a four-element ULA with different apertures under the illumination of two closely spaced emitters is studied. Then, ULAs with fixed apertures but different numbers of elements are considered. Lastly, DOA estimations of three closely spaced incoming signals using arrays with a fixed aperture but different numbers of elements are studied.

Case 1 (Four-Element ULAs with Different Apertures (2 Incoming Signals)). A ULA with four ideal isotropic sensors is studied. The objective is to investigate how the accuracy of DOA estimation is affected by the reduction of the array aperture. The separation of the array elements is changed from $\Delta=0.5 \lambda$ down to $0.05 \lambda$, with a step size of $0.05 \lambda$. This corresponds to a reduction of the array aperture (defined as the physical length of the array) from $1.5 \lambda$ to $0.15 \lambda$. The case with $\Delta=0.5 \lambda$ is the usual "standard" interelement separation used in those conventional arrays.

Two equal-power and equal-phase coherent sources come from the azimuth angles of $\phi_{1}=5^{\circ}$ and $\phi_{2}=6^{\circ}$, which are close to the endfire direction ( $\phi=90^{\circ}$ corresponds to the broadside direction). The signals (both magnitude and phase) are contaminated with AGWN at a signal-to-noise ratio (SNR) of $5 \mathrm{~dB}$. To evaluate the performance of the DOA estimations, the average bias, $\bar{b}$, is introduced and used as a metric throughout this paper. It is given by

$$
\bar{b}=\sum_{i=1}^{Q} \frac{b_{i}}{Q}
$$

where $b_{i}=\left|\widehat{\phi}_{i}-\phi_{i}\right|$ is the absolute value of the bias of the estimated direction $\left(\hat{\phi}_{i}\right.$ being the mean of the estimated directions; $\widehat{\phi}_{i}$ is determined from 10,000 independent Monte Carlo simulations), $Q$ is the number of incoming signals which is 2 in this case. For a successful DOA estimation, all the signals should be accurately estimated. A small value of average bias is obtained only if all the incoming signals are accurately estimated. At each value of interelement separation $(\Delta)$ shown in the figures in this paper, the average bias is obtained by 10,000 independent Monte Carlo simulations.

The results are shown in Figure 1. It is shown that the average biases decrease as the interelement separation increases. This indicates that the estimated directions are more accurate when the interelement separation is large. However, when $\Delta=0.05 \lambda$, the average bias is $3^{\circ}$ when the SNR levels of the two incoming signals are both $5 \mathrm{~dB}$. This means that MPM 


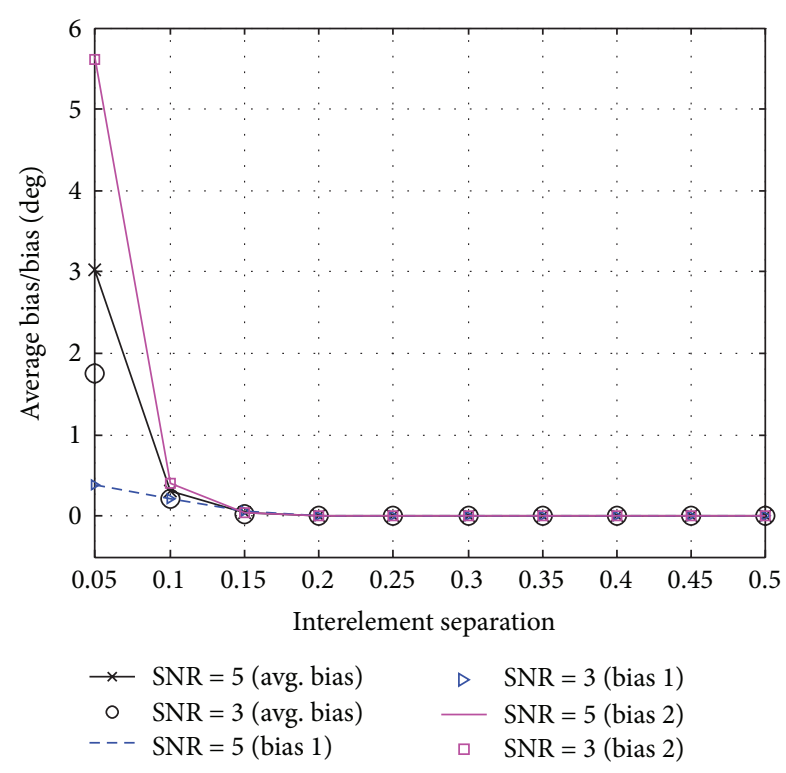

FIgURE 1: Summary of DOA estimations using MPM for a fourelement ULA with interelement separation varying from $0.05 \lambda$ to $0.5 \lambda$. Two equal-power incoming signals are from the directions of $\phi_{1}=5^{\circ}$ and $\phi_{2}=6^{\circ}$ with an SNR of $5 \mathrm{~dB}$ and $3 \mathrm{~dB}$, respectively. The graph shows (i) average bias of the estimated directions and (ii) bias of the estimation of signals 1 and 2 .

fails to resolve the two incoming signals as the difference between the two signals is only $1^{\circ}$. To probe further, the biases of the estimated directions of the two signals are also included. It is interesting to see that for the case of $\Delta=0.05 \lambda$, the bias of $\phi_{1}$ is about $0.4^{\circ}$ (i.e., the estimated direction is about $5.4^{\circ}$ ), which is close to the mean of the two incoming signals $\left(5^{\circ}\right.$ and $\left.6^{\circ}\right)$. The bias of $\phi_{2}$ is around $6^{\circ}$ which is far from the actual incoming direction, which further confirms that MPM fails to resolve the two incoming signals under such an array configuration. To investigate if the performance varies at different SNR level, the same DOA problem is repeated except that the $\mathrm{SNR}$ level is $3 \mathrm{~dB}$. It is found that there is no significant difference as compared to the case of SNR = $5 \mathrm{~dB}$.

Next, we study the effect on the performance of MPM when the incoming signals are not of equal power and equal phase. The entire procedure is repeated for the DOA estimation of $\phi_{1}=5^{\circ}$ and $\phi_{2}=6^{\circ}$ except that the amplitudes of the two signals are different and they are $90^{\circ}$ out of phase. For $\phi_{2}=6^{\circ}$, four different amplitudes of $A_{2}=2 A_{1}, 3 A_{1}, 5 A_{1}$, and $10 A_{1}$ are considered, where $A_{1}$ and $A_{2}$ are the amplitudes of the incoming signals at $\phi_{1}=5^{\circ}$ and $\phi_{2}=6^{\circ}$, respectively. The amplitude of the signal from $\phi_{1}=5^{\circ}$ remains to be the same for all cases. The average biases for the four cases are shown in Figure 2(a). The average biases in the range of $1.5^{\circ}$ to $2^{\circ}$ are found when $\Delta=0.05 \lambda$, which is considered as a fail estimate as the difference between the two incoming signals is only $1^{\circ}$. As $\Delta$ is larger than $0.2 \lambda$, the average biases decrease to the level of $10^{-3 \circ}$ which agrees with the results for the equalpower and equal-phase case shown in Figure 1. The biases of the two signals are also included in Figure 2(b). At $\Delta=0.05 \lambda$, the biases of $\phi_{1}=5^{\circ}$ and $\phi_{2}=6^{\circ}$ are at the levels of $0.5^{\circ}$ and $3^{\circ}$, respectively, which is similar to the results for the two signals with equal power in Figure 1. Such biases indicate that the two signals cannot be accurately resolved with this array aperture.

To investigate the performance of MPM for DOA estimations when the incoming signal direction changes, DOA estimation problems with the two signals coming from the directions of $\phi_{1}=10^{\circ}$ to $90^{\circ}$ and $\phi_{2}=\phi_{1}+1^{\circ}$ are considered. Here, only the angular range of $0^{\circ}$ to $90^{\circ}$ is considered as the angular range of $90^{\circ}$ to $180^{\circ}$ is simply the mirror image of that of $0^{\circ}$ to $90^{\circ}$. The same DOA estimation calculation procedure is repeated for all cases independently. The step size of $\Delta \phi_{1}=5^{\circ}$ is considered, and the results are shown in Figure 3. Similarly, the average biases decrease as $\Delta$ increases for the same incoming signals. The results show that the average bias is below $10^{-3 \circ}$ when the array element separation is greater than or equal to $0.2 \lambda$. Furthermore, we also note from the figure that as the signals are coming from a direction closer to the broadside direction of $90^{\circ}$, the accuracy of estimation increases quite significantly even when the element separation remains the same. For instance, when the element separation is $0.05 \lambda$ with incoming signals of $\phi_{1}=10^{\circ}$ and $\phi_{2}=11^{\circ}$, an average bias of $\sim 0.7^{\circ}$ is resulted. When the incoming signals become $\phi_{1}=55^{\circ}$ and $\phi_{2}=56^{\circ}$, the average bias now becomes $<10^{-4 \circ}$ which is considered as accurate (with less than $0.01 \%$ error).

Case 2 (Fixed Aperture ULAs with Different Numbers of Elements (2 Incoming Signals)). In the previous case, when the two closely spaced emitters approach to the endfire directions, it was found that the array aperture of the fourelement ULA has to be $\geq 0.60 \lambda(\Delta \geq 0.2 \lambda)$ for an accurate DOA estimation. Figure 4 shows the results of DOA estimations with different fixed array apertures that ranged from $0.25 \lambda$ to $1 \lambda$, but with a different number of array elements varying from 4 to 20 . Note that under a fixed array aperture, the interelement separation has to be changed with the change in the number of elements in the array. For example, for the four-element array with an aperture of $0.5 \lambda$, the interelement separation is $0.1667 \lambda(=0.5 \lambda /(4-1))$.

The array apertures of $0.25 \lambda$ and $0.5 \lambda$ are first considered, and the results are shown in Figures 4(a) and 4(b). When the incoming signals are close to the endfire directions (between $5^{\circ}$ and $15^{\circ}$ ), the average biases decrease when the number of elements increases from 4 to 20 . As the incoming signals are moving towards the broadside direction (between $20^{\circ}$ and $90^{\circ}$ ), the average biases decrease rapidly with an average bias less than $10^{-4 \circ}$ regardless of the number of elements.

Now, the same estimation procedure is repeated with an array aperture of $0.75 \lambda$. When the aperture is increased from $0.5 \lambda$ to $0.75 \lambda$, as shown in Figure $4(\mathrm{c})$, the average biases have been significantly reduced for all cases (below $2.2 \times 10^{-3 \circ}$ for all cases). The maximum average bias is reduced from $1.56 \times$ $10^{-2 \circ}(0.5 \lambda$ aperture $)$ to $2.2 \times 10^{-3 \circ}(0.75 \lambda$ aperture $)$, which is almost a factor of 7 . This indicates a significant improvement of the DOA estimations compared with the two cases of aperture size of $0.25 \lambda$ and $0.5 \lambda$. 


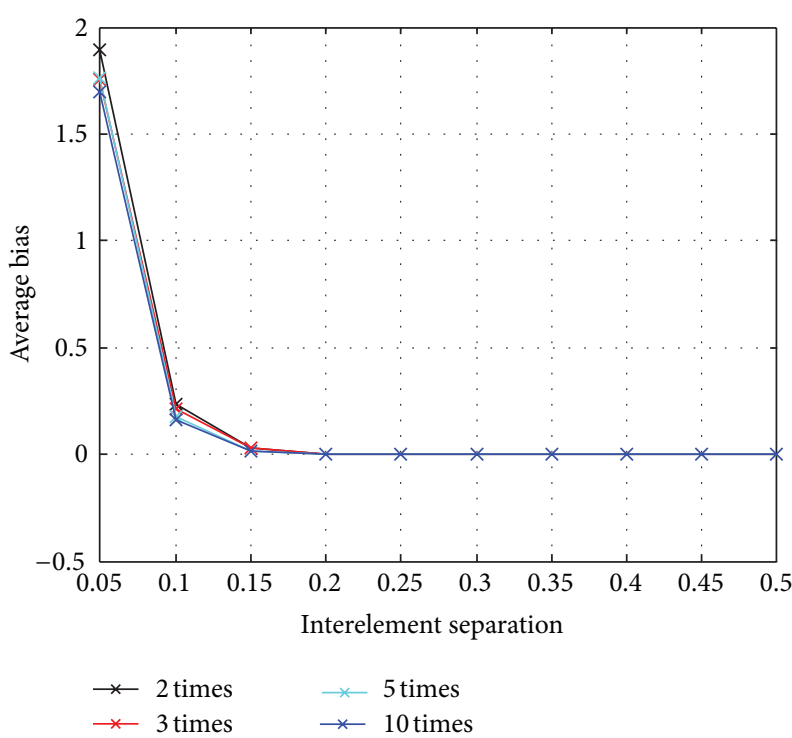

(a) Average Bias

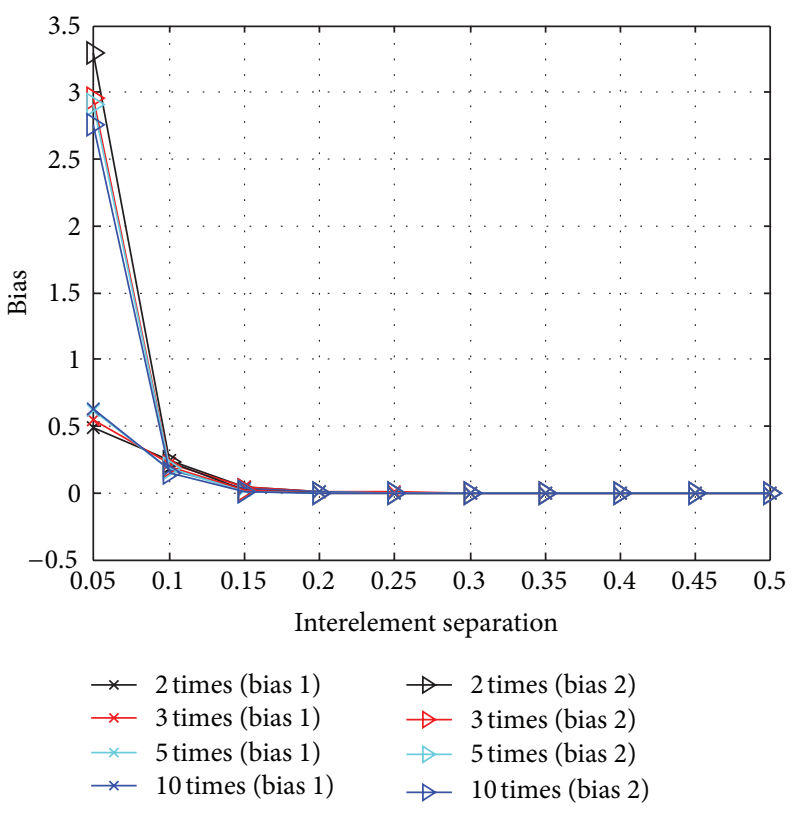

(b) Bias of Signal 1 and 2

FIgURE 2: Summary of DOA estimations using MPM for a four-element ULA with interelement separation varying from $0.05 \lambda$ to $0.5 \lambda$. The two incoming signals are from the directions of $\phi_{1}=5^{\circ}\left(A_{1}=1, \gamma_{1}=90^{\circ}\right)$ and $\phi_{2}=6^{\circ}\left(A_{2}=2,3,5\right.$, and $\left.10, \gamma_{2}=180^{\circ}\right)$ with an SNR of $5 \mathrm{~dB}$. (a) Average biases of the estimated directions; (b) biases of the estimation of signal 1 and signal 2.

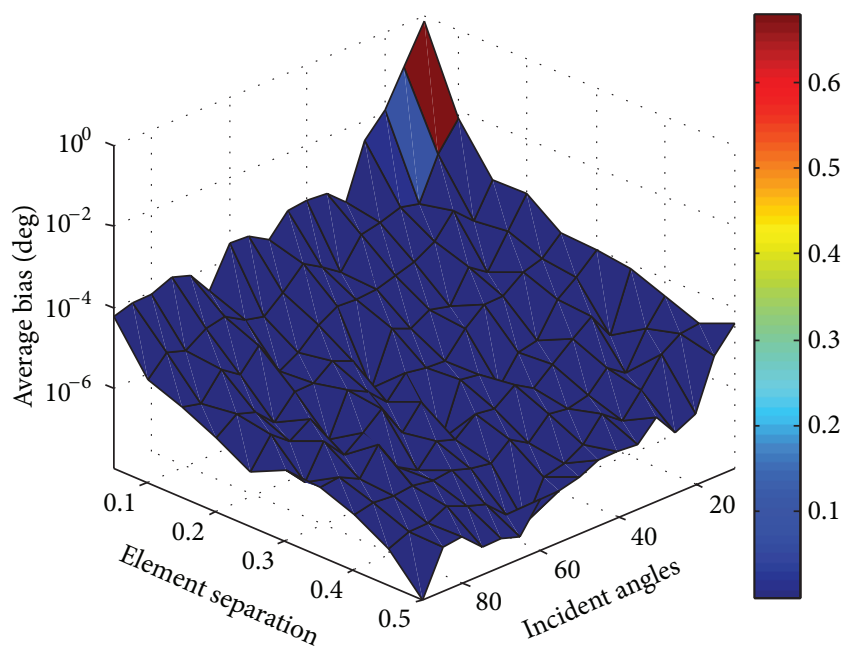

FIGURE 3: Summary of DOA estimations using MPM for a fourelement ULA with interelement separation varying from $0.05 \lambda$ to $0.5 \lambda$. Two equal-power incoming signals are from the directions of $\phi_{1}=10^{\circ}$ to $90^{\circ}$ and $\phi_{2}=\phi_{1}+1^{\circ}$ with an SNR of $5 \mathrm{~dB}$. The step size of $\Delta \phi_{1}=5^{\circ}$ is considered. The figure shows the average biases of the estimated directions.

The previous results show that for a fixed small array aperture (especially for the cases of $0.25 \lambda$ and $0.5 \lambda$ ), the accuracies of DOA estimation can be improved by increasing the number of elements when the incoming signals are close to the endfire directions. When the incoming signals are moving towards to the broadside direction, increasing the number of elements under a fixed aperture does not necessary improve the accuracies of the estimation. Comparing between signals coming from the broadside direction and the endfire directions, better accuracies are expected under the same array configuration.

When the array aperture is further increased to $1 \lambda$, as shown in Figure 4(d), it is not obvious if increasing the number of elements enhances the DOA performance under this aperture. On the other hand, it is worth noting that as the array aperture increases from $0.25 \lambda$ to $1 \lambda$, the average biases for all cases (different number of elements and incoming signals) decrease. This indicates that the accuracy of DOA estimation depends more on the array aperture than on the number of elements when the aperture is sufficiently large. While not presented here, the results on the array apertures of $1.25 \lambda$ and $1.5 \lambda$ indicate that there is some improvement of DOA accuracy as the number of elements increases. These results show that further increase in the number of elements for large aperture arrays $(>1 \lambda)$ only yields minor improvement to the accuracy of the estimated angles.

Case 3 (Fixed Aperture ULAs with Different Number of Elements (3 Incoming Signals)). In the last case, we consider DOA estimation problems of three closely spaced, equalpower, and equal-phase signals with an SNR of $5 \mathrm{~dB}$ come from the directions from $\phi_{1}=45^{\circ}$ to $90^{\circ}$ with $\Delta \phi_{1}=5^{\circ}$, $\phi_{2}=\phi_{1}+1^{\circ}$, and $\phi_{3}=\phi_{1}+2^{\circ}$. First, the array aperture of $0.75 \lambda$ is investigated. The number of array elements varies from 6 to 20 (the minimum number of sensors required for resolving 3 incoming signals using MPM is 6). The average biases of the DOA estimations are shown in Figure 5(a). It 


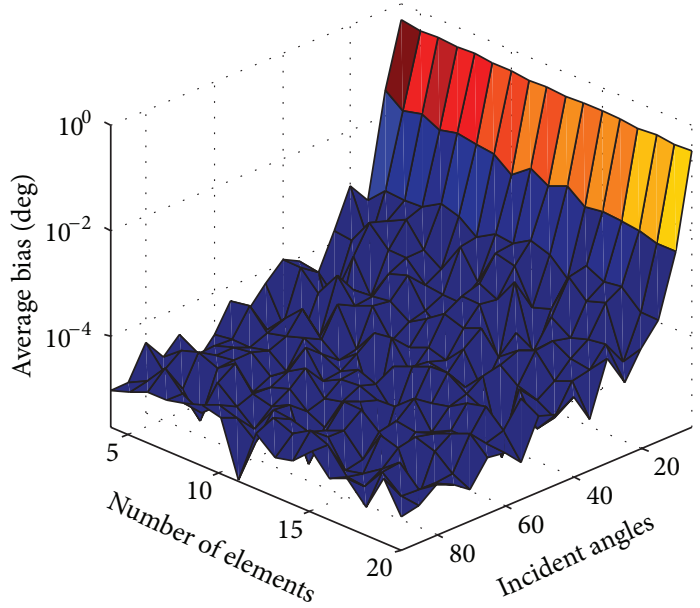

(a)

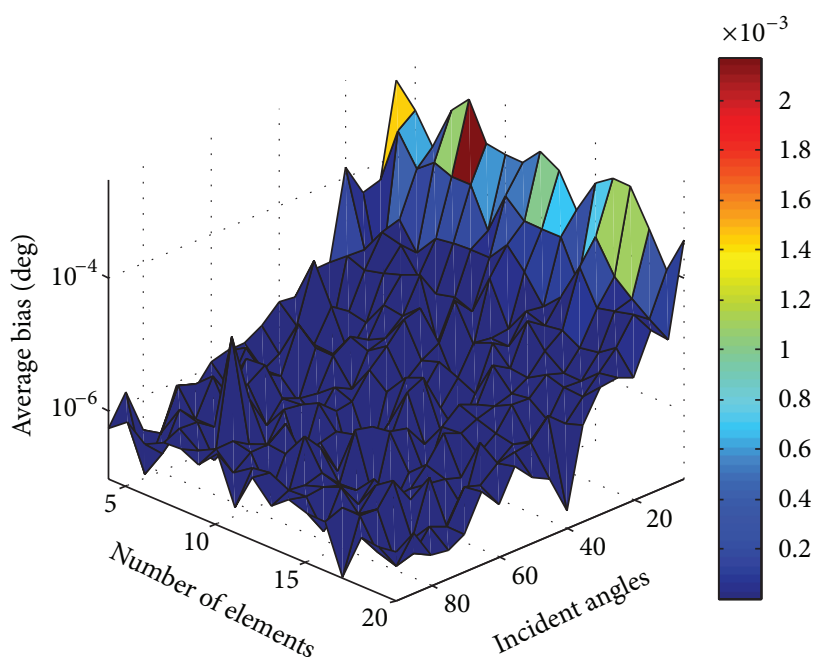

(c)
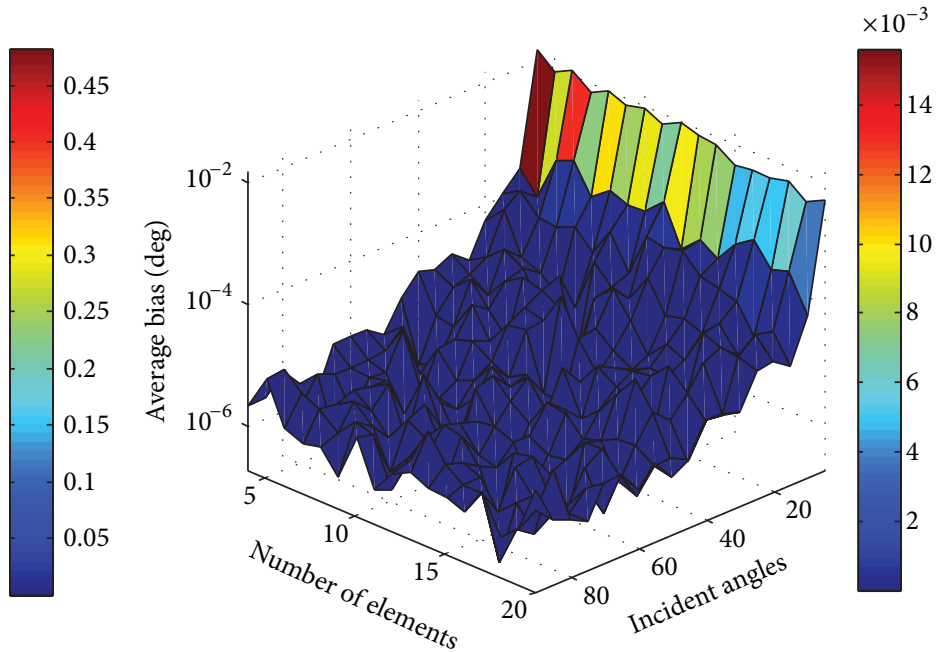

(b)

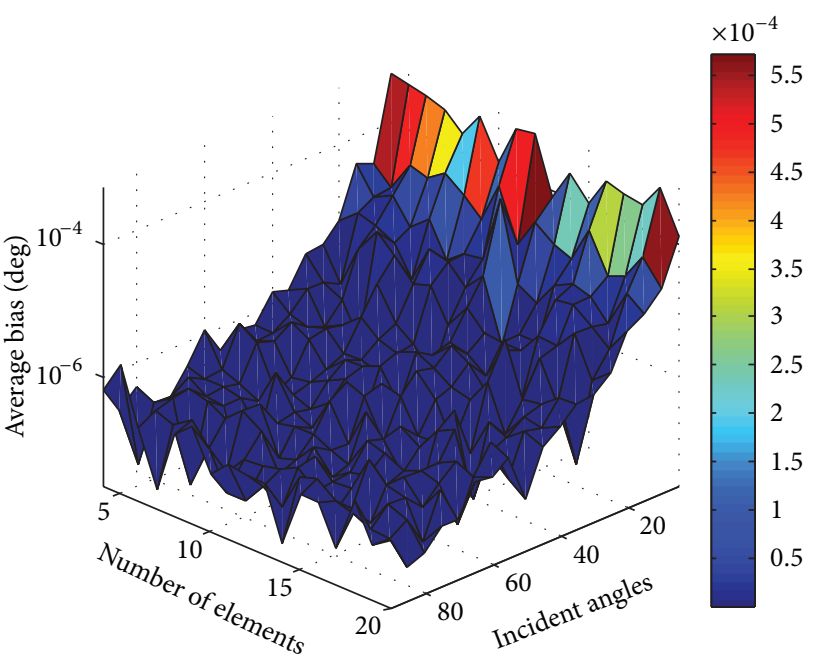

(d)

FIgURE 4: Summary of DOA estimations using MPM for a four-element ULA with the number of array elements varying from 4 to 20 . Two equal-power incoming signals are from the directions of $\phi_{1}=10^{\circ}$ to $90^{\circ}$ and $\phi_{2}=\phi_{1}+1^{\circ}$ with an SNR of $5 \mathrm{~dB}$. The step size of $\Delta \phi_{1}=5^{\circ}$ is considered. The figures show the average biases of the estimated direction. The apertures of the arrays are (a) $0.25 \lambda$, (b) $0.5 \lambda$, (c) $0.75 \lambda$, and (d) $1 \lambda$.

can be clearly seen that the average biases decrease with the number of the elements. Compared to the case with two signals of $\phi_{1}=45^{\circ}, \phi_{2}=46^{\circ}$ with the array aperture of $0.25 \lambda$ (Figure 4(a)) and the case of $\phi_{1}=5^{\circ}, \phi_{2}=6^{\circ}$ with the array aperture of $0.75 \lambda$ (Figure $4(\mathrm{c})$ ) with 6 array elements, the average biases presented in Figure 5(a) $\left(6^{\circ}\right)$ are much higher than those in Cases 1 and $2\left(10^{-4 \circ}\right.$ and $10^{-3 \circ}$, resp.). This is because resolving three incoming signals using MPM and resolving two incoming signals are two different mathematical problems with different CRLB, and they should be considered separately. As expected, when the incoming signals are moving towards the broadside directions, the average biases reduce to $2^{\circ}$ for the case of 6 antenna elements. The average biases are also reduced as the number of elements increases. However, even with 20 elements, the average biases are between $0.5^{\circ}$ and $3^{\circ}$. These bias values are considered as high as the actual separations between the three sources, only $1^{\circ}$, and thus it cannot be concluded that the sources are clearly resolved.

Lastly, the incoming signals of the same DOA problems and the array aperture are increased to $1 \lambda$ and $1.25 \lambda$, and the average biases are shown in Figures 5(b) and 5(c), respectively. For the same DOA problem, the average biases decrease as the number of array element increases. At an array aperture of $1 \lambda$ with 20 array elements, the average biases are at a level less than $0.1^{\circ}$ for DOA when the incoming signals are more than $75^{\circ}$. For the DOA problem with incoming signals $\left(\phi_{1}=45^{\circ}, \phi_{2}=46^{\circ}\right.$, and $\left.\phi_{3}=47^{\circ}\right)$ with 20 array elements, the average bias is $0.628^{\circ}$. However, when the array aperture is further increased to $1.25 \lambda$, the average bias reduces to smaller than $0.1^{\circ}$ with the 20 array elements (as shown in Figure 5(c)). The average biases for the same DOA estimation problem 


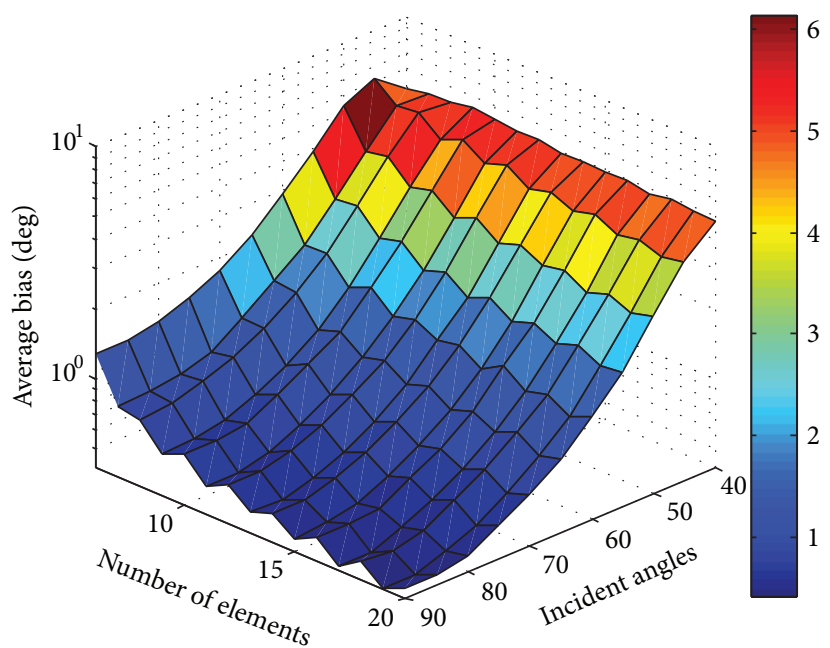

(a)

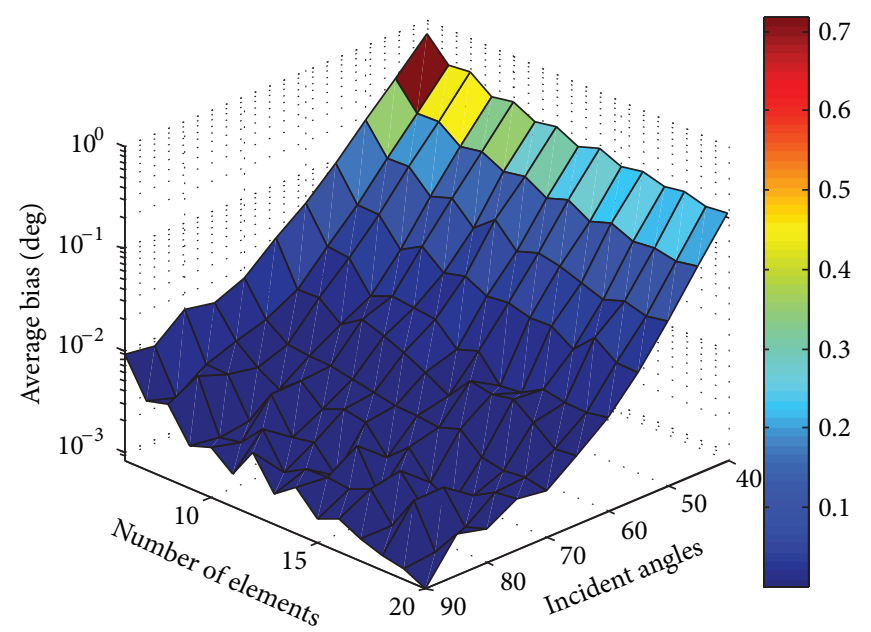

(c)

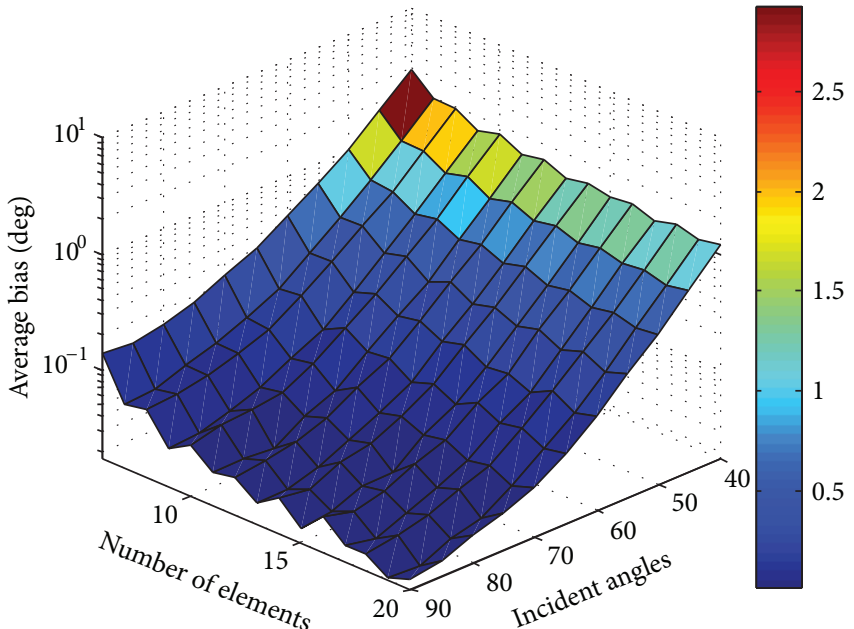

(b)

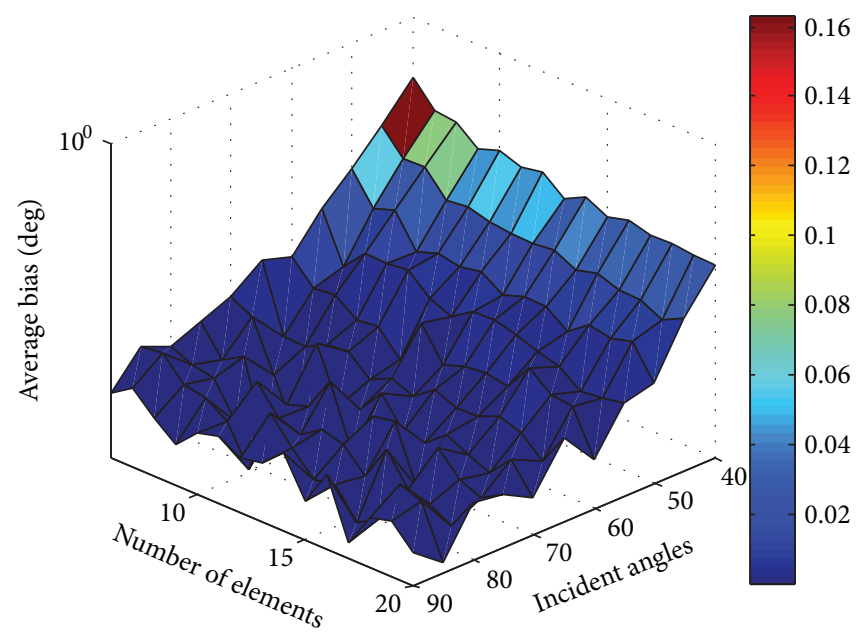

(d)

FIGURE 5: Summary of DOA estimations using MPM for a four-element ULA with the number of array elements varying from 6 to 20 . Three equal-power incoming signals are from the directions of $\phi_{1}=40^{\circ}$ to $90^{\circ}, \phi_{2}=\phi_{1}+1^{\circ}$, and $\phi_{3}=\phi_{1}+2^{\circ}$ with an SNR of $5 \mathrm{~dB}$. The step size of $\Delta \phi_{1}=5^{\circ}$ is considered. The figures show the average biases of the estimated directions with array aperture (a) $0.75 \lambda$, (b) $1.00 \lambda$, (c) $1.25 \lambda$, and (d) $1.50 \lambda$.

but with an array aperture of $1.5 \lambda$ are shown in Figure 5(d). As can be seen, when the signal sources are closer to the broadside direction, the average biases are smaller. However, an increasing number of array elements do not necessarily increase the accuracy of DOA estimation in this aperture. On the other hand, when the signal sources are closer to the endfire direction, the accuracy of DOA estimation does increase substantially when the number of array elements is increased (see Figure 5(d)).

\section{Discussions and Conclusions}

In this paper, we have investigated the performance of MPM in DOA estimations using ULAs with different array apertures and different numbers of array elements through Monte Carlo simulations. The results show that minimum linear array apertures of $0.6 \lambda$ and $1 \lambda$ are required to resolve two and three incoming signals, respectively (i.e., element separation of $\leq 0.2 \lambda$ ). The performance could be improved by increasing the number of elements. In practice, the emitters are less closely spaced which would be easier to resolve, and potentially, the size of the array and/or the number of elements can be further reduced. The finding provides us with the fundamental limit of DOA estimation in compact array. Arguably, the conclusions from this study are not limited to DOA estimations using compact antenna arrays (with interelement separation of $0.25 \lambda$ [35] down to $0.125 \lambda$ [37]) with MPM but are also applicable to acoustic or sonar arrays, as well as to other subspace methods.

\section{Acknowledgments}

This work was supported in part by the National University of Singapore (NUS) under the Grant no. R-263-000-469-112 
and in part by the US ONR research fund under the Project no. 09PR03332-01.

\section{References}

[1] J. C. Liberti and T. S. Rappaport, Smart Antennas for Wireless Communications, IS-95 and Third Generation CDMA Applications, Prentice Hall, Upper Saddle River, NJ, USA, 1999.

[2] H. Krim and M. Viberg, "Two decades of array signal processing research: the parametric approach," IEEE Signal Processing Magazine, vol. 13, no. 4, pp. 67-94, 1996.

[3] L. C. Godara, "Application of antenna arrays to mobile communications, part II: beam-forming and direction-of-arrival considerations," Proceedings of the IEEE, vol. 85, no. 8, pp. 11951245, 1997.

[4] A. Paulraj, B. Ottersten, R. Roy, A. Swindlehrst, G. Xu, and K. Kailath, "Subspace methods for directions-of-arrival esitmation," in Handbook of Statistics, vol. 10, chapter 16, pp. 693-739, Elsevier Science, Amsterdam, The Netherlands, 1993.

[5] R. O. Schmidt, "Multiple emitter location and signal parameter estimation," IEEE Transactions on Antennas and Propagation, vol. 34, no. 3, pp. 276-280, 1986.

[6] B. Friedlander and A. J. Weiss, "Direction finding in the presence of mutual coupling," IEEE Transactions on Antennas and Propagation, vol. 39, no. 3, pp. 273-284, 1991.

[7] A. L. Swindlehurst and T. Kailath, "A performance analysis of subspace-based methods in the presence of model errorspart I: the MUSIC algorithm," IEEE Transactions on Signal Processing, vol. 40, no. 7, pp. 1758-1774, 1992.

[8] D. Madurasinghe, "Super resolution technique for source power and bearing estimation," Electronics Letters, vol. 39, no. 17, pp. 1232-1233, 2003.

[9] A. Paulraj, R. Roy, and T. Kailath, "Subspace rotation approach to signal parameter estimation," Proceedings of the IEEE, vol. 74, no. 7, pp. 1044-1046, 1986.

[10] R. Roy and T. Kailath, "ESPRIT-estimation of signal parameters via rotational invariance techniques," IEEE Transactions on Acoustics, Speech, and Signal Processing, vol. 37, no. 7, pp. 984995, 1989.

[11] T. K. Sarkar and O. Pereira, "Using the matrix pencil method to estimate the parameters of a sum of complex exponentials," IEEE Antennas and Propagation Magazine, vol. 37, no. 1, pp. 4855, 1995.

[12] L. H. S. Antony and N. Shuley, "Consequence of incorrect sampling procedures in resonance-based radar target identification," Electronics Letters, vol. 40, no. 8, pp. 507-508, 2004.

[13] H. S. Lui and N. V. Z. Shuley, "Sampling procedures for resonance based radar target identification," IEEE Transactions on Antennas and Propagation, vol. 56, no. 5, pp. 1487-1491, 2008.

[14] H. Ouibrahim, D. D. Weiner, and T. K. Sarkar, "Generalized approach to direction finding," IEEE Transactions on Acoustics, Speech, and Signal Processing, vol. 36, no. 4, pp. 610-612, 1988.

[15] C. K. E. Lau, R. S. Adve, and T. K. Sarkar, "Minimum norm mutual coupling compensation with applications in direction of arrival estimation," IEEE Transactions on Antennas and Propagation, vol. 52, no. 8, pp. 2034-2041, 2004.

[16] T. K. Sarkar, M. C. Wicks, M. Salazar-Palma, and R. J. Bonneau, Smart Antennas, John Wiley \& Sons, 2003.

[17] Y. Hua, "Estimating two-dimensional frequencies by matrix enhancement and matrix pencil," IEEE Transactions on Signal Processing, vol. 40, no. 9, pp. 2267-2280, 1992.
[18] T. K. Sarkar, M. Salazar-Palma, and E. L. Mokole, Physics of Multiantenna Systems and Broadband Processing, John Wiley, 2008.

[19] S. Hwang, S. Burintramart, T. K. Sarkar, and S. R. Best, "Direction of Arrival (DOA) estimation using electrically small tuned dipole antennas," IEEE Transactions on Antennas and Propagation, vol. 54, no. 11, pp. 3292-3301, 2006.

[20] N. Yilmazer, J. Koh, and T. K. Sarkar, "Utilization of a unitary transform for efficient computation in the matrix pencil method to find the direction of arrival," IEEE Transactions on Antennas and Propagation, vol. 54, no. 1, pp. 175-180, 2006.

[21] Y. Hua and T. K. Sarkar, "Matrix pencil method for estimating parameters of exponentially damped/undamped sinusoids in noise," IEEE Transactions on Acoustics, Speech, and Signal Processing, vol. 38, no. 5, pp. 814-824, 1990.

[22] Y. Hua and T. K. Sarkar, "Generalized pencil-of-function method for extracting poles of an EM system from its transient response," IEEE Transactions on Antennas and Propagation, vol. 37, no. 2, pp. 229-234, 1989.

[23] N. Yilmazer, S. Burintramart, and T. K. Sarkar, "Performance analysis of direct data domain approach and ESPRIT method for DOA estimation," in Proceedings of the IEEE Antennas and Propagation Society International Symposium (AP-S '07), pp. 5295-5298, Honolulu, Hawaii, USA, June 2007.

[24] S. M. Kay, Fundamentals of Statistical Signal Processing: Estimation Theory, Prentice Hall, 1993.

[25] Y.-X. Yao and S. M. Pandit, "Cramer-Rao lower bounds for a damped sinusoidal process," IEEE Transactions on Signal Processing, vol. 43, no. 4, pp. 878-885, 1995.

[26] P. Stoica and A. Nehorai, "MUSIC, maximum likelihood, and Cramer-Rao bound," IEEE Transactions on Acoustics, Speech, and Signal Processing, vol. 37, no. 5, pp. 720-741, 1989.

[27] W. M. Steedly and R. L. Moses, "Cramer-Rao bound for pole and amplitude coefficient estimates of damped exponential signals in noise," IEEE Transactions on Signal Processing, vol. 41, no. 3, pp. 1305-1318, 1993.

[28] E. H. Djermoune and M. Tomczak, "Perturbation analysis of subspace-based methods in estimating a damped complex exponential," IEEE Transactions on Signal Processing, vol. 57, no. 11, pp. 4558-4563, 2009.

[29] K. R. Dandekar, H. Ling, and G. Xu, "Experimental study of mutual coupling compensation in smart antenna applications," IEEE Transactions on Wireless Communications, vol. 1, no. 3, pp. 480-487, 2002.

[30] H. T. Hui, "Improved compensation for the mutual coupling effect in a dipole array for direction finding," IEEE Transactions on Antennas and Propagation, vol. 51, no. 9, pp. 2498-2503, 2003.

[31] H.-S. Lui, H. T. Hui, and M. S. Leong, "A note on the mutualcoupling problems in transmitting and receiving antenna arrays," IEEE Antennas and Propagation Magazine, vol. 51, no. 5, pp. 171-176, 2009.

[32] H. S. Lui and H. T. Hui, "Improved mutual coupling compensation in compact antenna arrays," IET Microwaves, Antennas and Propagation, vol. 4, no. 10, pp. 1506-1516, 2010.

[33] H. S. Lui and H. T. Hui, "Mutual coupling compensation for direction-of-arrival estimations using the receiving-mutualimpedance method," International Journal of Antennas and Propagation, vol. 2010, Article ID 373061, 2010.

[34] Y. Yu, H. S. Lui, C. H. Niow, and H. T. Hui, "Improved DOA estimations using the receiving mutual impedances for mutual 
coupling compensation: an experimental study," IEEE Transactions on Wireless Communications, vol. 10, no. 7, pp. 2228-2233, 2011.

[35] C. Volmer, J. Weber, R. Stephan, K. Blau, and M. A. Hein, "An eigen-analysis of compact antenna arrays and its application to port decoupling," IEEE Transactions on Antennas and Propagation, vol. 56, no. 2, pp. 360-370, 2008.

[36] B. K. Lau, J. B. Anderson, G. Kristensson, and A. F. Molisch, "Impact of matching network on bandwidth of compact antenna arrays," IEEE Transactions on Antennas and Propagation, vol. 54, no. 11, pp. 3225-3237, 2006.

[37] D. M. Browne, M. Manteghi, M. P. Fitz, and Y. R. Samii, "Experiments with compact antenna arrays for MIMO radio communications," IEEE Transactions on Antennas and Propagation, vol. 54, no. 11, pp. 3239-3250, 2006. 

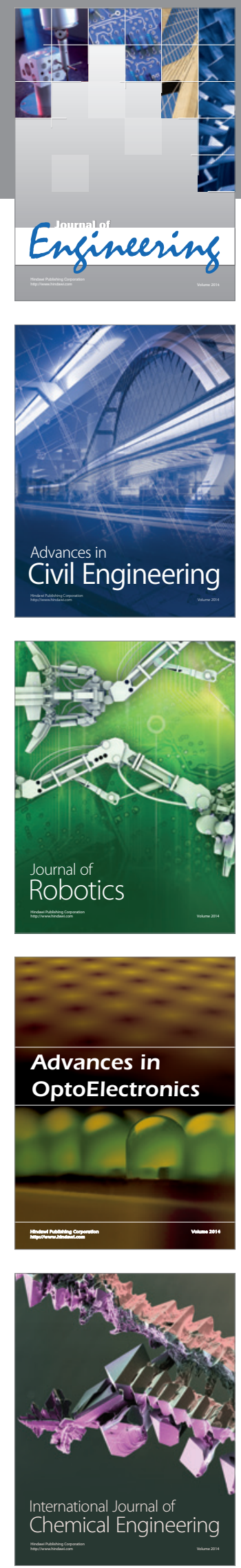

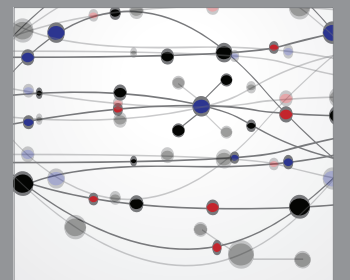

The Scientific World Journal
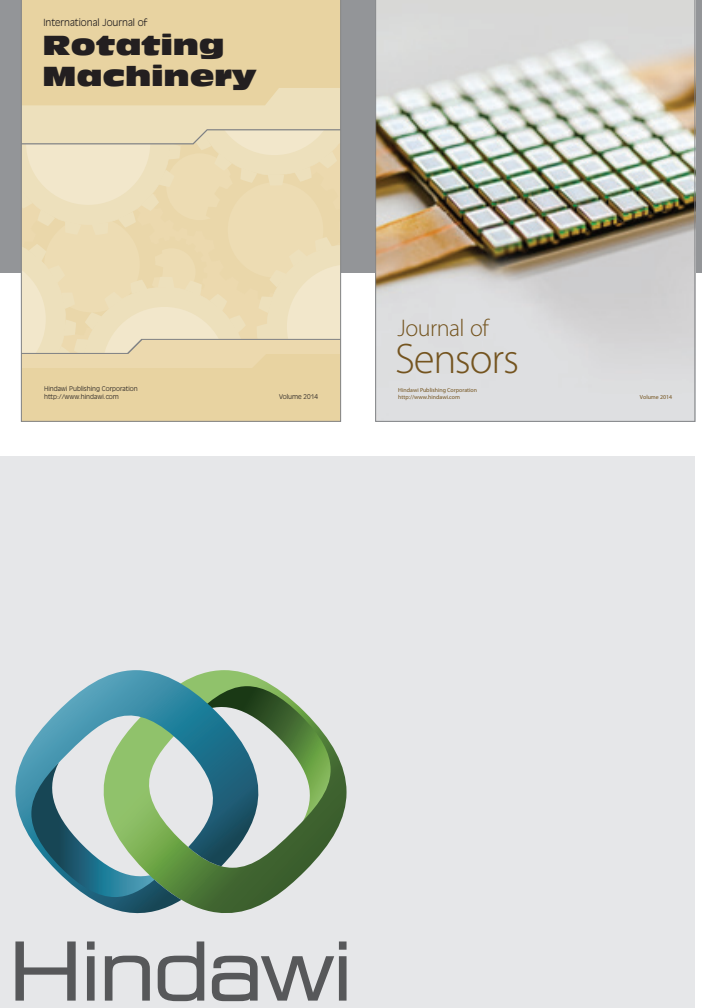

Submit your manuscripts at http://www.hindawi.com
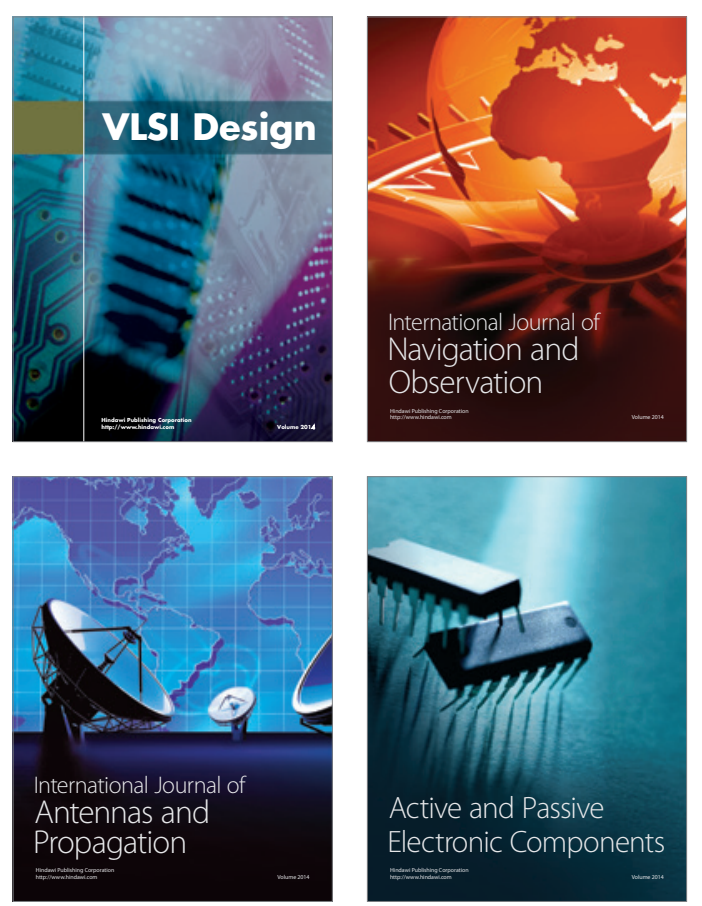
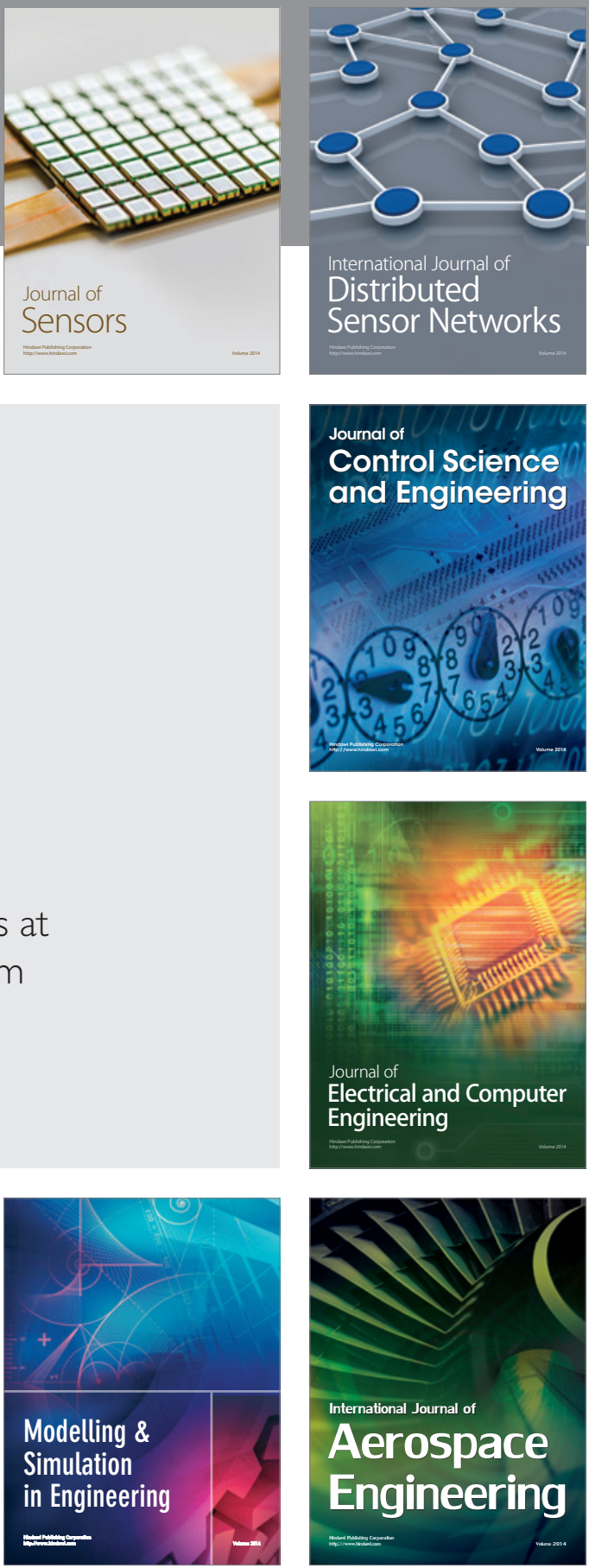

Journal of

Control Science

and Engineering
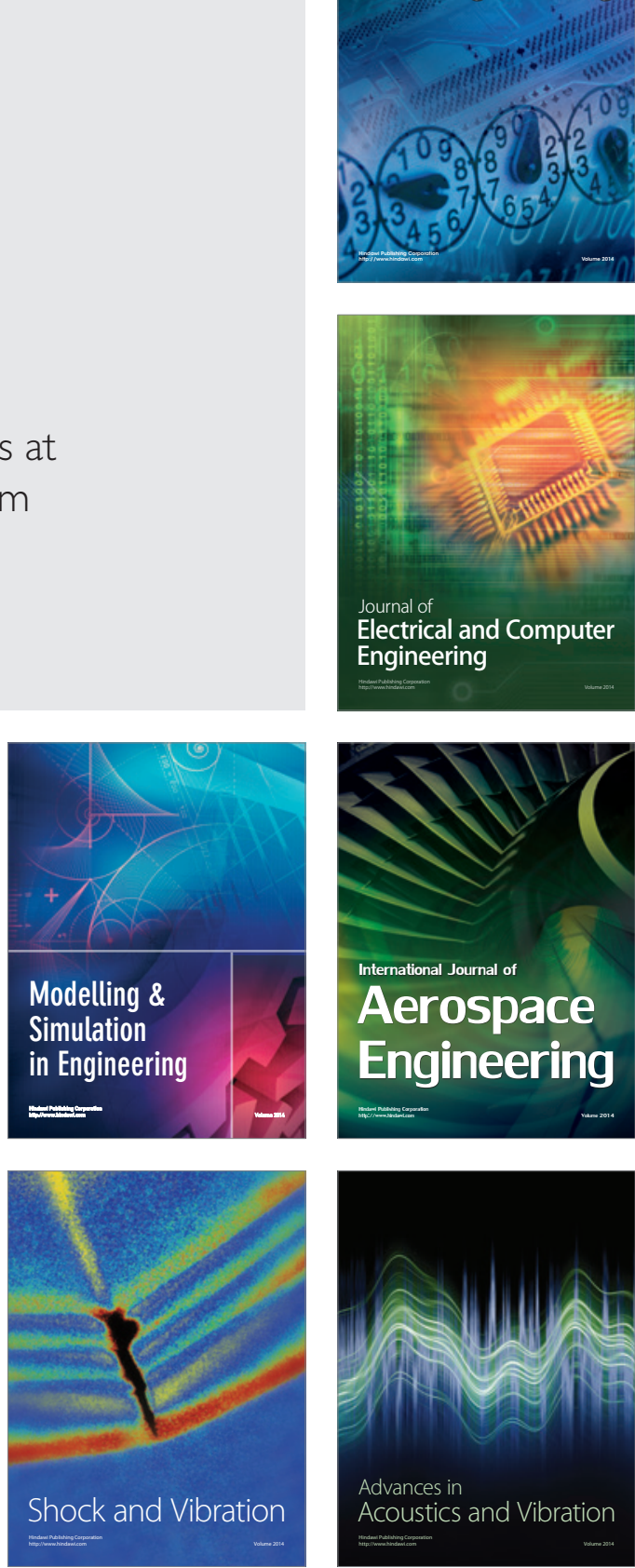